\title{
Parental access to hospitalised children during infectious disease pandemics such as COVID-19
}

\author{
A Goga, ${ }^{1,2}$ FC Paed (SA), PhD; U Feucht, ${ }^{2,3,4,5}$ FC Paed (SA), PhD; S Pillay, ${ }^{6}$ FC Paed (SA), Cert Neonatology (SA); \\ G Reubenson, ${ }^{7}$ FC Paed (SA); P Jeena, ${ }^{8}$ FC Paed (SA), PhD; S Madhi, ${ }^{9,10}$ FC Paed (SA), PhD; N T Mayet, ${ }^{11}$ MB ChB; \\ S Velaphi, ${ }^{7}$ FC Paed (SA), PhD; N McKerrow, ${ }^{6,8,12}$ FC Paed (SA); L R Mathivha, ${ }^{13}$ PGDHSE; N Makubalo, ${ }^{14}$ FC Paed (SA); \\ R J Green, ${ }^{2}$ FC Paed (SA), DSc; G Gray, ${ }^{1}$ FC Paed (SA), DSc \\ ${ }^{1}$ South African Medical Research Council, Cape Town, South Africa \\ ${ }^{2}$ Department of Paediatrics and Child Health, School of Medicine, Faculty of Health Sciences, University of Pretoria, South Africa \\ ${ }^{3}$ Tshwane District Health Services, Gauteng Department of Health, City of Tshwane, South Africa \\ ${ }^{4}$ Research Centre for Maternal, Fetal, Newborn and Child Health Care Strategies, School of Medicine, Faculty of Health Sciences, University of \\ Pretoria, South Africa \\ ${ }^{5}$ Maternal and Infant Health Care Strategies Research Unit, South African Medical Research Council, Pretoria, South Africa \\ ${ }^{6}$ Department of Paediatrics and Child Health, Faculty of Health Sciences, University of Cape Town, South Africa \\ ${ }^{7}$ Department of Paediatrics and Child Health, Faculty of Health Sciences, University of the Witwatersrand, Johannesburg, South Africa \\ ${ }^{8}$ Department of Paediatrics and Child Health, College of Health Sciences, University of KwaZulu-Natal, Durban, South Africa \\ ${ }^{9}$ South African Medical Research Council: Vaccines and Infectious Diseases Analytics Research Unit, School of Pathology, Faculty of Health Sciences, \\ University of the Witwatersrand, Johannesburg, South Africa \\ ${ }^{10}$ Department of Science and Technology/National Research Foundation, South African Research Chair Initiative in Vaccine Preventable Diseases, \\ Faculty of Health Sciences, University of the Witwatersrand, Johannesburg, South Africa \\ ${ }^{11}$ National Institute of Communicable Diseases, Johannesburg, South Africa \\ ${ }^{12}$ Department of Paediatrics and Child Health, KwaZulu-Natal Department of Health, Pietermaritzburg, South Africa \\ ${ }^{13}$ Department of Critical Care Medicine, Faculty of Health Sciences, University of the Witwatersrand, Johannesburg, South Africa \\ ${ }^{14}$ District Clinical Specialist Team, Eastern Cape Department of Health, Nelson Mandela Bay, South Africa
}

Corresponding author: A Goga (ameena.goga@mrc.ac.za)

The COVID-19 pandemic has resulted in many hospitals severely limiting or denying parents access to their hospitalised children. This article provides guidance for hospital managers, healthcare staff, district-level managers and provincial managers on parental access to hospitalised children during a pandemic such as COVID-19. It: (i) summarises legal and ethical issues around parental visitation rights; (ii) highlights four guiding principles; (iii) provides 10 practical recommendations to facilitate safe parental access to hospitalised children; (iv) highlights additional considerations if the mother is COVID-19-positive; and ( $v$ ) provides considerations for fathers. In summary, it is a child's right to have access to his or her parents during hospitalisation, and parents should have access to their hospitalised children; during an infectious disease pandemic such as COVID-19, there is a responsibility to ensure that parental visitation is implemented in a reasonable and safe manner. Separation should only occur in exceptional circumstances, e.g. if adequate in-hospital facilities do not exist to jointly accommodate the parent/caregiver and the newborn/infant/child. Both parents should be allowed access to hospitalised children, under strict infection prevention and control (IPC) measures and with implementation of non-pharmaceutical interventions (NPIs), including handwashing/sanitisation, face masks and physical distancing. Newborns/infants and their parents/caregivers have a reasonably high likelihood of having similar COVID-19 status, and should be managed as a dyad rather than as individuals. Every hospital should provide lodger/boarder facilities for mothers who are COVID-19-positive, COVID-19-negative or persons under investigation (PUI), separately, with stringent IPC measures and NPIs. If facilities are limited, breastfeeding mothers should be prioritised, in the following order: (i) COVID-19-negative; (ii) COVID-19 PUI; and (iii) COVID-19-positive. Breastfeeding, or breastmilk feeding, should be promoted, supported and protected, and skin-to-skin care of newborns with the mother/caregiver (with IPC measures) should be discussed and practised as far as possible. Surgical masks should be provided to all parents/caregivers and replaced daily throughout the hospital stay. Parents should be referred to social services and local community resources to ensure that multidisciplinary support is provided. Hospitals should develop individual-level policies and share these with staff and parents. Additionally, hospitals should ideally track the effect of parental visitation rights on hospital-based COVID-19 outbreaks, the mental health of hospitalised children, and their rate of recovery.

S Afr Med J 2021;111(2):100-105. https://doi.org/10.7196/SAMJ.2021.v111i2.15388

During infectious disease pandemics such as COVID-19, there is often a dilemma as to whether hospitalised children should be separated from their parents: in many settings, health facilities have severely limited or denied parents access to their hospitalised children, and thus restricted hospitalised children's access to their parents. Childhood is a period of physical, psychological and social vulnerabilities, which may be exacerbated during illness. In the face of COVID-19, the physical, emotional and social repercussions associated with hospital admission may be amplified by the effects of social distancing, infection prevention and control 
(IPC) measures, and non-pharmaceutical interventions (NPIs) such as personal protective equipment (PPE). This article provides guidance for hospital managers, healthcare staff, district-level managers and provincial managers on parental access to hospitalised children during a pandemic such as COVID-19. It summarises legal and ethica issues around parental visitation rights, highlights four guiding principles, provides 10 practical recommendations to facilitate safe parental access to hospitalised children, highlights additional considerations if the mother is COVID-19-positive, and provides considerations for fathers.

Parental presence during hospitalisation has several benefits for both parent and child, including parental stress reduction as parents become more informed and involved in caring for their hospitalised children, leading to greater parental satisfaction. ${ }^{[1]}$ Systematic reviews have demonstrated that parents and healthcare providers recognise the value of parents being with their child during hospitalisation. ${ }^{[2,3]}$ This is especially pertinent to newborns, where kangaroo mother care (KMC) is associated with significantly shorter hospital stay, less frequent readmission and increased maternal satisfaction. ${ }^{[4]}$ In addition, skin-to-skin contact in the first hours of life is associated with reduced postpartum haemorrhage risk, decreased rates of postpartum depression and anxiety, and increased odds of successful breastfeeding. ${ }^{[5]}$

In the context of COVID-19, there has been rapid, continual assimilation of data quantifying the risks and outcomes of newborns born to mothers with COVID19. While individual reports have reported possible cases of intrauterine SARS-CoV-2 transmission, breastmilk transmission has not been described. However, horizontal transmission through respiratory spread is a recognised mode of transmission. Cluster outbreaks of COVID-19 have occurred in mothers' lodges and $\mathrm{KMC}$ units, associated with adverse outcomes. These have led to cautious expert opinions and individualised management principles, including predelivery counselling with temporary postdelivery separation of SARS-CoV-2-infected mothers from their infants, with expressed breastmilk feeding using strict infection control practices. ${ }^{[6,7]}$ However, the World Health Organization recommends that mother-infant pairs should remain together while rooming in throughout the day and night, practising skin-to-skin contact, including KMC, particularly in the immediate postpartum period and during establishment of breastfeeding, whether or not mothers or their infants have suspected or confirmed
COVID-19. ${ }^{[8]}$ This recommendation is also endorsed by the European Paediatric Association. ${ }^{[9]}$ Separation of mother-infant pairs could theoretically minimise the risk of SARS-CoV-2 transmission; however, at the time of writing this article, there was no compelling evidence suggesting benefit of separation. ${ }^{[10]}$ Additionally, separation may not prevent infection (as described in a case series from Wuhan), and it does not prevent infection after discharge. ${ }^{[6]}$ It could also disrupt newborn physiology (higher heart and respiratory rates, and lower glucose levels in newborns). ${ }^{[10]}$ As noted by the Royal College of Midwives, Obstetricians and Gynaecologists and Paediatricians in the UK, 'routine precautionary separation of a mother and a healthy baby should not be undertaken lightly, given the potential detrimental effects on feeding and bonding. ${ }^{\text {[11] }}$ Isolation is a significant stressor for newborns; for infants already infected with SARS-CoV-2, isolation could worsen the disease course. Separation also increases maternal stress levels, increasing heart rate and salivary cortisol levels, and in the context of a pandemic this additional suffering may worsen the mother's disease course. ${ }^{[10]}$ Current evidence shows that the risk of newborn infection is very low, and most infected newborns do not have significant morbidity. ${ }^{[5]}$ The benefit of KMC to the infant-mother dyad outweighs the risks, particularly in low- and middleincome countries. Consequently, the mother/ caregiver should be empowered and given the option to practise KMC with explanation of risks and benefits, with emphasis on IPC and NPIs, to reduce the risk of transmission. Infant-mother dyads should remain together

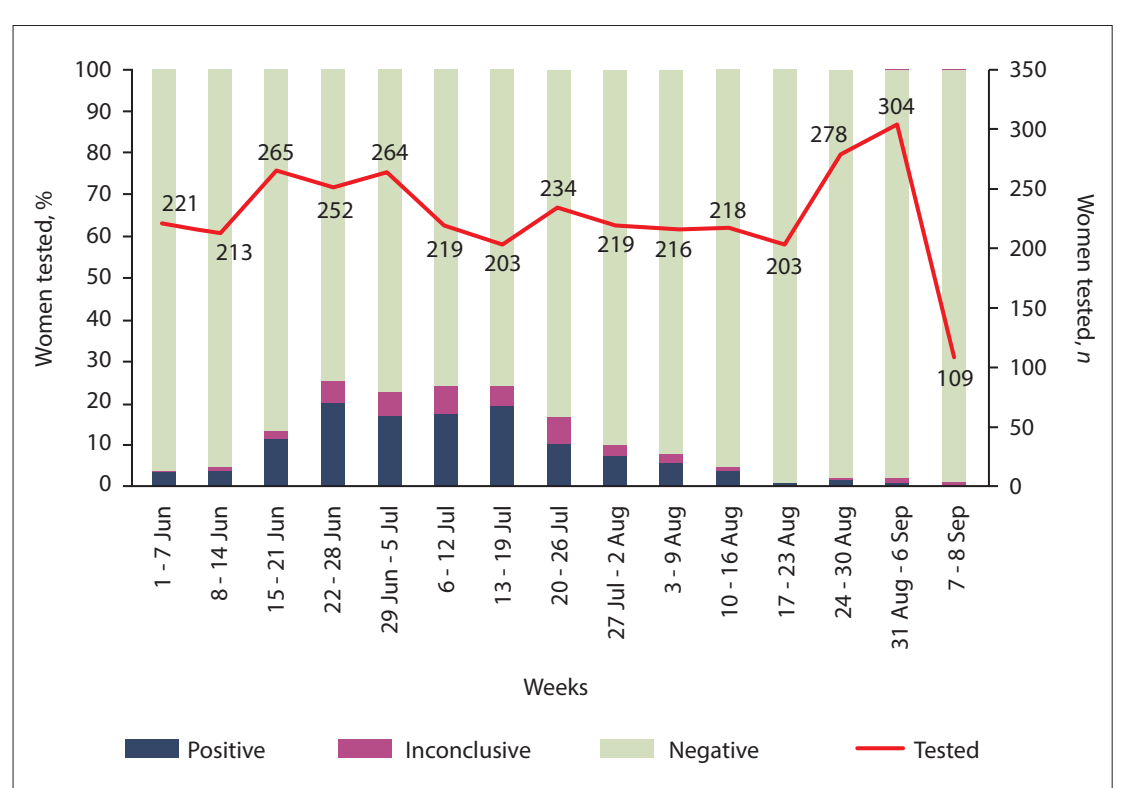

Fig. 1. SARS-CoV-2 testing and results in pregnant women at Chris Hani Baragwanath Hospital,

Johannesburg, 1 June - 8 September 2020 (source: Shabir Madhi).

if possible, and KMC should be encouraged; these infants do not need to be nursed in an incubator if they remain isolated with their mother - when not doing skin-to-skin they can be in a crib next to the mother's bed. The alternative, which is complete cessation of visitation during a pandemic, while not empirically studied, would undoubtedly add to parental stress and may have subsequent deleterious effects on infant development. ${ }^{[12]}$ Lastly, separation could interfere with maternal milk production and supply, disrupting feeding and innate and specific immune protection. ${ }^{[10]}$

\section{Experiences in the South African context and abroad}

In May 2020, despite IPC measures, 9 mothers, 2 babies, 4 doctors and a nurse tested positive for SARS-CoV-2 at the General Justice Gizenga Mpanza Regional Hospital (previously Stanger Hospital) in KwaZuluNatal Province, South Africa (SA). ${ }^{[13]}$ The source of infection was probably a lodger mother who had undisclosed close contact with a COVID-19 case. Similarly, in June 2020, a COVID-19 outbreak occurred in the KMC ward at Chris Hani Baragwanath Academic Hospital (CHBAH), Johannesburg, SA (S Velaphi, personal communication). Testing of pregnant women at $\mathrm{CHBAH}$ demonstrated that their COVID-19 positivity rates mirror the positivity rates in the community (Fig. 1). The effect of strict in-hospital IPC and NPI measures on COVID-19 positivity in maternal, child or KMC wards during the second wave remains to be measured, including whether patient positivity sparks hospital outbreaks. 
A Lancet Child and Adolescent Health publication reporting data from a New York hospital during March - May 2020 states that of the 120 babies born to 116 COVID-positive mothers, none had acquired SARS-CoV-2 by 1 month of age. ${ }^{[14]}$ All neonates who roomed in with their mothers were nursed in a closed Giraffe isolette (General Electric Healthcare, USA); skin-to-skin contact was initiated in the delivery room with appropriate IPC, and infants were held by their mothers during feeding after appropriate hand hygiene and breast cleansing, with maternal surgical mask use. All mothers were allowed to breastfeed. Mothers of neonates admitted to the specialised neonatal unit were only allowed to visit 14 days after they tested positive, if they had been afebrile for at least 72 hours.

\section{Legall rights and ethics around parental visitation rights}

As eloquently articulated by McQuoid-Mason, ${ }^{[15]}$ the ban on parental visitation to their hospitalised children is a violation of children's rights, as per the SA Constitution, and in terms of acting in the 'best interests of the child' and the 'best interests standard' (Children's Act No. 38 of 2005). He argues that the restrictions on parental visitation are not 'reasonable and justifiable' and that 'less restrictive means' can be used to prevent the spread of SARS-CoV-2. Hospital policies that limit visitors, and by extension limit mobility, are rooted in consequentialist ideals. ${ }^{[16]}$ This means that such limitations must have a positive impact on the greater constituency. It can be argued that restricting parental access to hospitalised children is entrenched within utilitarianism, i.e. maximising the good for the highest number of people, and libertarianism, which states that the the only purpose for which power can be rightfully exercised over any member of a civilized community against his will, is to prevent harm to another. ${ }^{\text {[17] }}$ The current pandemic justifies limiting liberties using utilitarianism and libertarianism and the draconian limitation of parents at the bedside only if there are no less intrusive measures of preventing the spread of SARS-CoV-2. In 1984, the Siracusa Principles, outlined by the United Nations, coalesced the conditions necessary to legitimise restrictive public health measures in the setting of a pandemic. ${ }^{[18]}$ Importantly, these principles stipulate that the least restrictive measures of interference and disruption should be used to achieve the public health goal. In the context of COVID19, the proper application of PPE can contain and reduce spread. ${ }^{[19,20]}$ However, the prospect of allowing asymptomatic carriers, who are potentially contagious, into any neonatal or paediatric unit is daunting. This anxiety stems from staff members and other mothers coming into contact with someone who is COVID-19-positive. Even with PPE a zero risk to others cannot be guaranteed, and therefore health services cannot guarantee 'first do no harm' for all. However, the risk of SARSCoV-2-positive mothers transmitting infection and causing severe illness in the baby has been low until now, so it is reasonable to keep infants with their mothers even if the mother is COVID-19-positive, provided she is well enough to care for her baby and follows strict IPC measures. Lastly, the ethics of contrasting hospital policies in the same subdistrict/local area (whereby some hospitals allow parental access while others do not) is disingenuous because the guiding principle is not based on facts particular to the case or the community, but instead on hospital preference. ${ }^{[17]}$

\section{Systems meeded to ensure parental access to hospitalised children during COVID-19}

Within the context of infectious disease outbreaks, such as the current COVID-19 pandemic, we recommend avoiding the separation of parents and hospitalised children. Newborns/infants/children and their parents/caregivers have a reasonably high likelihood of having a similar COVID-19 status. The child and parent should therefore be considered and managed as a dyad rather than as separate individuals. However, COVID-19-positive mothers may spread SARS-CoV-2 to other mothers or to hospital staff, so a positive SARS-CoV-2 result in either member of the dyad should prompt management of both as potentially infectious. Children should have access to both parents during their hospitalisation. This approach is guided by four principles:

- First do no physical, emotional or social harm. The best interests (nutrition, health, development and wellbeing) of every child should be prioritised. Children should not be harmed, and all decisions should not cause harm to other children, caregivers or staff.

- Maximise the good for the highest number of individuals, while causing no harm.

- Apply the least restrictive measures of interference and disruption to achieve the public health goal.

- Consider the feasibility of implementing recommended protocols in different social, cultural and geographical contexts, including settings with limited resources.

Consequently, we provide 10 recommendations (Box 1), namely: (i) avoid separation of the hospitalised child and his or her parents, except under exceptional circumstances; (ii) newborns/infants/children and their parents/caregivers are likely to have similar COVID-19 status and should be managed as a single dyad rather than as separate individuals; (iii) promote, support and encourage breastfeeding, or breastmilk feeding, and discuss skin-to-skin care of newborns with the

\section{Box 1. Ten recommendations to facilitate safe parental access to hospitalised children}

1. Avoid separation: Separation of parent/caregiver and newborn/infant/child should only occur under exceptional circumstances, e.g. in-hospital facilities do not exist to jointly accommodate them.

- To minimise the possibility of physical, emotional and social harm to young children, every child requiring admission to hospital should be accompanied by a parent/caregiver, based on the child's maturity and needs.

- As far as possible, every newborn/child needing hospitalisation should be admitted with his/her parent/caregiver.

- Both parents should be allowed access to hospitalised children, under strict IPC measures and with NPIs, including hand sanitisation, face masks and physical distancing.

- Parental counselling should include counselling of both mothers and fathers. One-on-one counselling should include what is expected from parents, if the dyad is positive, negative or unknown (see classifications below), IPC measures and NPIs including hand sanitisation, wearing masks and physical distancing.

- The parents/family should identify the primary person who will stay with the child, with the understanding that this primary person will remain with the child for the full duration of the hospital admission. If exceptional circumstances arise and the primary person is unable to stay for the full duration, a pass-out may be allowed, and parents may change over. Strict IPC measures and NPIs should be followed to prevent SARSCoV-2 infection or limit spread. 
Box 1. (continued) Ten recommendations to facilitate safe parental access to hospitalised children

- Current evidence shows that the risk of newborn infection is low, and most infected newborns do not have significant morbidity. The benefit from KMC to both members of the infant/mother dyad outweighs the risks, particularly in LMICs. Consequently, the mother/caregiver should be empowered, and in the case of newborns given the option to do KMC with an explanation of its risks and benefits, with emphasis on IPC and NPIs to reduce the risk of transmission. Mother-newborn dyads should remain together if possible, and KMC should be encouraged; these infants do not need to be nursed in an incubator if they remain with their mother in isolation. If not doing skin-to-skin, they can be in a cot next to the mother's bed.

2. Newborns/infants/children and their parents/caregivers are likely to have similar COVID-19 status and should be managed as a dyad rather than as separate individuals. Caregiver and child should be tested for COVID-19 for cohorting:

- COVID-19-negative dyad: both child AND caregiver have negative COVID-19 tests.

- COVID-19 PUI dyad: unknown COVID-19 status of child and/or caregiver.

- COVID-19-positive dyad: either child OR caregiver has a positive COVID-19 test.

Members of each category of dyad should be accommodated together, and every hospital needs to assess where to accommodate each category:

- COVID-19-negative dyads should be accommodated in the COVID-negative ward/unit (this is the preferred option - a lounger chair should be provided), or the mother may be accommodated away from the child in the COVID-19-negative mothers' lodge/cubicle/ward.

- COVID-19 PUI dyads should be accommodated in a designated unit/ward (this is the preferred option), or the mother may be accommodated in the COVID-negative mothers' lodge/cubicle/ward.

- COVID-19-positive dyads should be accommodated in the COVID-19 unit/ward (this is the preferred option), or the mother may be accommodated in a COVID-19-positive lodger/boarder facility or in an isolation field facility (with transport provided if the isolation facility is not on site).

- If facilities exist, rooming in is the best option.

- Large lodges can be split into sections if there are multiple ablution and social spaces: (i) COVID-19-negative; (ii) COVID-19 PUI; (iii) COVID19-positive.

- If space is limited, based on utilitarianism, libertarianism, the Siracusa principle and the four principles above, breastfeeding mothers should be prioritised, in the following order of preference: (i) COVID-19-negative; (ii) COVID-19 PUI; (iii) COVID-19-positive.

3. Promote, support and encourage breastfeeding, or breastmilk feeding, and discuss skin-to-skin care of newborns with the mother/caregiver. Ideally, promote breastfeeding and skin-to-skin contact, with IPC measures and NPIs (i.e. maternal surgical masks, hourly hand hygiene and respiratory etiquette). KMC units should continue if cohorting is possible, IPC and NPIs are maintained, and the physical space facilitates isolation of infected dyads with distancing from uninfected dyads. Individual health facilities may tailor local decisions after consultation with district-level and paediatric experts, based on local epidemic load, availability of space, IPC and NPIs. Individual healthcare providers may tailor skin-to-skin decisions for an individual dyad after counselling a mother/caregiver, based on their choice, and ability to implement IPC and NPIs.

4. Provide surgical masks to all parents/caregivers accompanying a child to hospital. These should be replaced daily throughout the hospital stay.

5. Every hospital is mandated to provide lodger/boarder facilities for mothers, with stringent IPC monitoring, surgical masks for all mothers, physical distancing, regular handwashing and twice-daily COVID-19 screening. The lodger facility should be well ventilated and without overcrowding.

6. Reinforce administrative controls to reduce risk:

- Stagger mealtimes of lodger mothers.

- Ring a bell hourly during waking hours to remind everyone about handwashing/sanitising in the ward/lodger facility.

- Identify an infection control marshal in each unit to:

- ensure physical distancing (at least $1 \mathrm{~m}$ and preferably $2 \mathrm{~m}$, with a mask on) in the lodger facility/KMC unit/neonatal unit/paediatric medical and surgical ward

- ensure adequate ventilation while keeping mothers/caregivers/babies warm

- monitor/prevent close contact between staff and mothers/caregivers during mealtimes.

- Identify a 'champion' to orientate new lodgers and oversee implementation of and compliance with IPC measures and NPIs:

- enforce strict hand hygiene - hourly handwashing or use of alcohol-based hand rub during waking hours

- enforce the wearing of face masks by all lodgers at all times, except when bathing, eating or sleeping

- eat outside, weather permitting, or in a well-ventilated area, away from other lodgers (preferably $2 \mathrm{~m}$ apart, and at least $1 \mathrm{~m}$ apart)

- implement 12-hourly symptom screening of all lodgers

- restrict lodgers' movement to walking between their accommodation and their child's ward.

- Provide surgical masks to all parents/caregivers on entrance to the hospital, with daily replacement thereof throughout the hospital stay.

- Provide sanitiser at every bed/incubator/bassinet.

- Inform each parent/caregiver about the risk of COVID-19 during their hospital stay.

7. Reinforce engineering controls to reduce risk:

- Space beds in the mother's lodge at least $1 \mathrm{~m}$ but preferably $2 \mathrm{~m}$ apart.

- Space hospital beds/incubators/bassinets at least $1 \mathrm{~m}$ but preferably $2 \mathrm{~m}$ apart.

8. If parental separation is unavoidable, it should be limited to as short a period as possible, and innovative methods should be implemented to facilitate contact, including daily phone calls, photographs and video calls, and skin-to-skin interactions by a caregiver or staff member designated to care for the newborn/infant/child. This skin-to-skin should occur only if caregiver/staff member is wearing a clean surgical mask, has sanitised his/her hands and observes cough etiquette. Assistance with lactation/re-lactation after the acute illness should be standard of care.

9. Engage with communities to explore the repurposing of homes/community halls around the hospital to accommodate dyads.

10. Link parents to social services and local community resources and/or facilitate access to grants, as policies and practices (e.g. IPC measures/NPIs) around parental access to hospitalised children during COVID-19 could cause or exacerbate stress. 
mother/caregiver; (iv) provide surgical masks to all parents/caregivers accompanying a child to hospital, with daily replacement thereof throughout the hospital stay; $(v)$ every hospital is mandated to provide lodger/boarder facilities for mothers, with stringent IPC monitoring, surgical masks, physical distancing, regular handwashing and twice daily COVID-19 screening - the lodger facility should be well ventilated and not overcrowded; ( $v i)$ reinforce administrative controls to reduce risk; (vii) reinforce engineering controls to reduce risk; (viii) if parental separation is unavoidable, it should be limited to as short a period as possible, and innovative methods should be implemented to facilitate contact, including daily phone calls, photographs and video calls, and skin-to-skin interactions by a caregiver or staff member designated to care for the newborn/infant/child; (ix) engage with communities to explore the repurposing of homes/community halls around the hospital to accommodate dyads; and $(x)$ link parents to social services and/or local community resources, as policies and practices (e.g. IPC measures/NPIs) concerning parental access to hospitalised children during COVID-19 could cause or exacerbate stress.

Box 2. Additional considerations when a mother is COVID-19-positive

Scenario 1. Mother and newborn/infant/child are well

- Do not hospitalise. Discharge newborn/infant/child to isolate at home with mother.

- Promote and support breastfeeding.

- Encourage skin-to-skin in newborns if the mother is able to don a clean surgical mask just before commencing skin-to-skin contact, has sanitised her hands and observes cough etiquette.

- Both to isolate for 10 days (or as per latest NICD recommendations).

- Parents to follow advice to prevent SARS-CoV-2 transmission to the newborn/infant/child, including hand hygiene, cough etiquette, mask wearing, and cleaning of breasts before breastfeeding.

- Emphasise importance of continued breastfeeding and routine care during and after isolation.

Scenario 2. Mother needs hospitalisation

- Scenario 2A. Hospital facilities exist - breastfeeding newborns and infants who are not mobile (not crawling/walking) may remain with mother in isolation:

- Mother to wear a surgical mask and to observe all IPC measures and NPIs as outlined above.

- Promote and support breastfeeding.

- Mother should provide skin-to-skin contact for her newborn if she is able to wear a clean surgical mask, has sanitised her hands and observes cough etiquette. The mother should interact with the newborn/infant (touch, speak) regularly.

- Discharge as soon as possible.

- Both to isolate for 10 days (or as per latest NICD guidelines).

- Provide advice to prevent SARS-CoV-2 transmission to the newborn/infant, including the importance of hand hygiene, cough etiquette, mask wearing, and cleaning of breasts before breastfeeding.

- Emphasise importance of continued breastfeeding and routine care, including immunisations, during and after isolation.

- Scenario 2B. There are no or limited facilities, or IPC cannot be maintained - separation is unavoidable: newborn/infant/child will be discharged into the care of father/caregiver:

- Mother to remain in hospital only for as long as absolutely necessary and to be discharged as soon as possible.

- Promote and support breastfeeding and encourage breastmilk feeding at home: mother should express milk and father/caregiver should collect milk and take it home, observing strict IPC and handwashing/breast and container sanitisation measures. Alternatively, the hospital may assist with obtaining donor breastmilk, if feasible.

- While the dyad is separated, the father/caregiver should provide skin-to-skin contact and interact with the newborn/infant/child (touch, speak) regularly. Skin-to-skin should occur only if father/caregiver is wearing a clean surgical mask, has sanitised his/her hands and observes cough etiquette.

- Both to isolate for 10 days (or as per latest NICD recommendations).

- Use innovative methods to keep mother in contact with newborn/infant/child. Send mother photographs or videos or call mother using video calls to keep mother-baby pair in touch with each other during this difficult time.

- Counsel on importance of breastfeeding and routine care once the 10-day period has been completed.

- Support the mother to re-lactate/resume breastfeeding when reunited with baby/infant.

Scenario 3. Newborn baby/infant/child needs hospitalisation

- Scenario 3A. Hospital isolation facilities exist - mother can remain with newborn baby/infant/child:

- Follow guidance as per scenario $2 \mathrm{~A}$ above.

- Scenario 3B. There are no lodger/boarder facilities or IPC cannot be maintained - separation is unavoidable:

- Newborn/infant/child to remain in hospital only for as long as absolutely necessary and to be discharged as soon as possible.

- Promote and support breastfeeding and encourage breastmilk feeding: mother should express milk and father/caregiver should deliver milk, observing strict IPC and handwashing with breast and container sanitisation measures. Alternatively, the hospital may assist with obtaining donor breastmilk, if feasible.

- While the dyad is separated, hospital staff should interact with the baby (touch, speak) regularly. Skin-to-skin should occur only if the staff member is wearing a clean surgical mask, has sanitised her/his hands and observes cough etiquette.

- Mother and newborn/infant/child to isolate for 10 days (or as per latest NICD guidelines).

- Use innovative methods to keep mother in contact with newborn/infant/child: send mother photographs or videos or call mother using video calling to keep mother-baby pair in touch with each other during this difficult time.

- Counsel on importance of breastfeeding and routine care once the 10-day isolation period has been completed.

- Support mother to re-lactate/resume breastfeeding when reunited with baby/infant.

NICD = National Institute for Communicable Diseases; IPC = infection prevention and control; NPIs = non-pharmacological interventions 
Box 3. Special considerations for fathers

Fathers must be allowed to visit their hospitalised children, with unrestricted access and the following provisos:

- As with mothers, fathers must be provided with a surgical mask before entering the ward/unit.

- IPC measures and NPIs should be used at all times. These include hand sanitisation, face-mask wearing and physical distancing from staff/ other caregivers/other children.

- When a child is critically ill, during end-of-life care and/or when both parents are required for counselling, fathers must be allowed access.

IPC $=$ infection control and prevention; NPIs = non-pharmacological interventions

We highlight additional considerations if the mother is COVID19-positive (Box 2), and special considerations for fathers (Box 3 ).

Hospitals should develop individual-level policies guided by evidence, feasibility, experience and ethical principles of utilitarianism and libertarianism, and share these with staff and parents. Additionally, hospitals should ideally track the effect of parental visitation rights on hospital-based COVID-19 outbreaks, the mental health of hospitalised children, and their rate of recovery.

\section{Conclusions}

It is a child's right to have access to his or her parents during hospitalisation, and parents should have access to their hospitalised children, but there is a responsibility to ensure that this is done in a reasonable and safe manner. During infectious disease pandemics, such as COVID-19, the coverage of NPIs and IPC measures must be expanded to facilitate reasonable access of parents to their hospitalised children. The impact of this on hospital-based COVID19 outbreaks, the mental health of hospitalised children, and their rate of recovery should be monitored.

\section{Declaration. None.}

Acknowledgements. South African Medical Research Council.

Author contributions. AG: conceptualised article, wrote drafts incorporated comments, finalised drafts. UF: contributed reviews and papers, contributed to the discussions, assisted with summarising consensus and main points, reviewed all drafts and the final document. SP: contributed reviews and papers, contributed to the discussions, reviewed all drafts and the final document. GR: reviewed all drafts, contributed to the discussions and assisted with consensus. PJ: reviewed and contributed to all drafts and the final document. SM: reviewed and contributed to all drafts and the final document, and contributed the data on testing in pregnant women. NM: contributed to all drafts and reviewed the final document. SV: reviewed all drafts and the final document and contributed experience from a local setting. NMcK: reviewed all drafts and the final document and contributed to the discussions. LRM: reviewed all drafts and the final document and contributed to the discussions. NM: reviewed all drafts and the final document and contributed to the discussions. RJG: reviewed all drafts and the final document. GG: assisted with conceptualisation of the article, and reviewed all drafts and the final document.

Funding. South African Medical Research Council.

\section{Conflicts of interest. None.}

1. Melnyk B, Alpert-Gillis L, Feinstein N, et al. Creating opportunities for parent empowerment: Program effects on the mental health/coping outcomes of critically ill young children and their mothers. Pediatrics 2004;113(6):e597-607. https://doi.org/10.1542/PEDS.113.6.E597

2. Shields L, Zhou H, Pratt J, Taylor M, Hunter J, Pascoe E. Family-centred care for hospitalised children aged 0 - 12 years. Cochrane Database Syst. Rev 2012, Issue 10. Art. No.: CD004811. https:// doi.org/10.1002/14651858.CD004811.pub3

3. Watts R, Zhou H, Taylor M, Munns A, Ngune I. Family-centered care for hospitalized children aged $0-12$ years: A systematic review of qualitative studies. JBI Database System Rev Implement Rep 2014;12(7):204-283. https://doi.org/10.11124/jbisrir-2014-1683

4. Jafari M, Farajzadeh F, Asgharlu Z, Derakhshani N, Asl YP. Effect of kangaroo mother care on Jafari M, Farajzadeh F, Asgharlu Z, Derakhshani N, Asl YP. Effect of kangaroo mother care on
hospital management indicators: A systematic review and meta-analysis of randomized controlled trials. J Educ Health Promot 2019;8:96. https://doi.org/10.4103/jehp.jehp_310_18

5. Boscia C. Skin-to-skin care and COVID-19. Pediatrics 2020;146(2):e20201836. https://doi. org/10.1542/peds.2020-1836

6. Zeng, L, Xia S, Yuan W, et al. Neonatal early-onset infection with SARS-CoV-2 in 33 neonates born to mothers with COVID-19 in Wuhan, China. JAMA Pediatr 2020;174(7):722-725. https://doi. org/10.1001/jamapediatrics.2020.0878

7. De Carvalho WB, Gibelli MABC, Krebs VLJ, Calil VMLT, Johnston C. Expert recommendations for the care of newborns of mothers with COVID-19. Clinics 2020;75(2):e1932. https://doi.org/10.6061/ clinics/2020/e1932

8. World Health Organization. Breastfeeding and COVID-19, Scientific Brief. 23 June 2020. https:// www.who.int/news-room/commentaries/detail/breastfeeding-and-covid-19 (accessed 10 October 2020).

9. Williams J, Namazova-Baranova L, Weber M, et al. The importance of continuing breastfeeding during coronavirus disease-2019: In support of the World Health Organization Statement on Breastfeeding during the Pandemic. J Pediatr 2020;223:234-236. https://doi.org/10.1016/j. jpeds.2020.05.009

10. Stuebe A. Should infants be separated from mothers with COVID-19? First, do no harm. Breastfeed Med 2020;15(5):351-352. https://doi.org/10.1089/bfm.2020.29153.ams

11. Royal College of Midwives, Royal College of Obstetricians and Gynaecologists. Coronavirus (COVID-19) infection in pregnancy: Information for health care professionals. Version II. 24 July 2020. https://www.rcog.org.uk/globalassets/documents/guidelines/2020-07-24-coronavirus-covid19-infection-in-pregnancy.pdf (accessed 10 October 2020).

12. Turpin H, Urben S, Ansermet F, Borghini A, Murray MM, Müller-Nix C. The interplay between prematurity, maternal stress and children's intelligence quotient at age 11: A longitudinal study. Sci Rep 2019;9:450. https://doi.org/10.1038/s41598-018-36465-2

13. Duma N. Two babies, 14 adults test positive for COVID-19 at KZN hospital. Eye Witness News, 5 May 2020. https://ewn.co.za/2020/05/05/two-babies-14-adults-test-positive-for-covid-19-in-kznhospital (accessed 10 October 2020)

14. Salvatore C, Han J-Y, Acker KP, et al. Neonatal management and outcomes during the COVID-19 pandemic: An observation cohort study. Lancet Child Adolesc Health 2020;4(10):721-727. https:// doi.org/10.1016/S2352-4642(20)30235-2

15. McQuoid-Mason DJ. Is the COVID-19 regulation that prohibits parental visits to their children who are patients in hospital invalid in terms of the Constitution? What should hospitals do? S Afr Med J 2020;110(11):1089-1087. https://doi.org/10.7196/SAMJ.2020.v110i11.15273

16. Wynia M. Ethics and public health emergencies: Restrictions on liberty. Am J Bioeth 2007;7(2):1-5. https://doi.org/10.1080/15265160701577603

17. Murray P, Swanson J. Visitation restrictions: Is it right and how do we support families in the NICU during COVID-19? J Perinatol 2020;40:1576-1581. https://doi.org/10.1038/s41372-020-00781-1

18. United Nations. Siracusa Principles on the Limitation and Derogation Provisions in the International Covenant on Civil and Political Rights. 28 September 1984. https://undocs.org/pdf?symbol=en/E/ CN.4/1985/4 (accessed 29 August 2020).

19. Cook TM. Personal protective equipment during the coronavirus disease (COVID) 2019 pandemic - a narrative review. Anaesthesia 2020;75(7):920-927. https://doi.org/10.1111/anae.15071

20. Chu DK, Akl EA, Duda S, et al. Physical distancing, face masks, and eye protection to prevent person-to-person transmission of SARS-CoV-2 and COVID-19: A systematic review and metaanalysis. Lancet 2020;395(10242):1973-1987. https://doi.org/10.1016/S0140-6736(20)31142-9 